\title{
A novel blood brain barrier-permeable IRE1 kinase inhibitor sensitizes glioblastoma to chemotherapy in mice.
}

Diana Pelizzari-Raymundo ${ }^{1,2,8}$, Dimitrios Doultsinos ${ }^{1,2,8,10}$, Raphael Pineau ${ }^{1,2}$, Chloé Sauzay ${ }^{1,2}$, Thodoris Koutsandreas ${ }^{3,4}$, Antonio Carlesso ${ }^{5}$, Elena Gkotsi ${ }^{3,4}$, Luc Negroni $^{6}$, Tony Avril ${ }^{1,2}$, Aristotelis Chatziioannou ${ }^{3,4}$, Xavier Guillory ${ }^{1,2,7,9}$, Leif A. Eriksson ${ }^{5,9}$, Eric Chevet ${ }^{1,2,9}$

${ }^{1}$ INSERM U1242, Université de Rennes, Rennes, France. ${ }^{2}$ Centre de Lutte contre le Cancer Eugène Marquis, Rennes, France. ${ }^{3}$ e-NIOS PC, Kallithea-Athens, Greece. ${ }^{4}$ Center of Systems Biology, Biomedical Research Foundation of the Academy of Athens, Athens, Greece. ${ }^{5}$ Department of Chemistry and Molecular Biology, University of Gothenburg, Göteborg, Sweden. ${ }^{6}$ Institut de Génétique et de Biologie Moléculaire et Cellulaire, Centre National de la Recherche Scientifique, UMR7104, Institut National de la Santé et de la Recherche Médicale, U1258, Université de Strasbourg, 67404 Illkirch, France. ${ }^{7}$ Institut des Science Chimiques de Rennes, CNRS UMR6226, Université de Rennes, Rennes, France.

Keywords: Endoplasmic Reticulum • IRE1 • Inhibitors - glioblastoma • Unfolded Protein Response

Running title: New IRE1 inhibitors in GB

${ }^{8} \mathrm{DPR}$ and DD equally contributed to this work

${ }^{9}$ Equally contributed and corresponding authors EC eric.chevet@inserm.fr - LAE leif.eriksson@chem.gu.se-XG xavier.guillory@univ-rennes1.fr

${ }^{10}$ Present address: Nuffield Department of Surgical Sciences, John Radcliffe Hospital, University of Oxford, Oxford OX3 9DU, UK. 


\section{Abstract}

Inositol Requiring Enzyme 1 (IRE1) is a bifunctional serine/threonine kinase and endoribonuclease. It is a major mediator of the Unfolded Protein Response (UPR), which is activated during endoplasmic reticulum (ER) stress. Tumor cells experience ER stress due to adverse microenvironmental cues such as hypoxia or nutrient shortage and high metabolic/protein folding demand. To cope with those stresses, cancer cells utilize IRE1 signaling as an adaptive mechanism. Here we report the discovery of novel IRE1 inhibitors identified through a structural exploration of the IRE1 kinase domain. We first characterized these candidates in vitro and in cellular models. We showed that all molecules inhibit IRE1 signaling and sensitize glioblastoma cells to the standard chemotherapeutic temozolomide (TMZ). From these inhibitors, we retained a Blood-Brain Barrier (BBB) permeable molecule (Z4P) and demonstrated its ability to inhibit Glioblastoma (GB) growth and to prevent relapse in vivo when administered together with TMZ. These results support the attractiveness of IRE1 as an adjuvant therapeutic target in GB. We thus satisfy an unmet need for targeted, nontoxic, IRE1 inhibitors as adjuvant therapeutic agents against GB. 


\section{Introduction}

In recent years the activation of the Unfolded Protein Response (UPR) by the PKRlike ER kinase (PERK), the activating transcription factor 6 (ATF6) and the Inositol requiring enzyme 1 alpha (IRE1 $\alpha$, referred to as IRE1 hereafter) has been demonstrated to play an instrumental role in cancer development and aggressiveness. From these three main ER stress sensors that transduce the UPR from the ER lumen to the nucleus, the potential of IRE1 activity inhibition as a therapeutic intervention in multiple disease states has been the subject of extensive investigation $(1,2)$. The activity of IRE1 depends on both its kinase and ribonuclease domains to produce its functional output. The ATP binding kinase domain facilitates IRE1 phosphorylation that in turn allows for the conformational change that activates the RNase domain. The RNase domain then channels the functional output of IRE1 by unconventionally cleaving XBP1 mRNA to produce XBP1s, a potent transcription factor, or by cleaving a subset of mRNAs and miRNAs in a process called RIDD (RNA IRE1 Dependent Decay). Inhibiting IRE1 activity can thus be achieved by compounds targeting either the ATP-binding kinase pocket or the RNase domain. Studies have demonstrated that Kinase inhibiting RNase attenuators (KIRAs) may have an inhibitory effect on IRE1 RNase activity $(3,4)$. Other IRE1 kinase inhibitors indirectly inhibit the RNase by impeding IRE1 phosphorylation (5). Lastly, IRE1 RNase inhibitors include $4 \mu 8 \mathrm{c}$, STF083010, and a series of salicylaldehyde MKC compounds including MKC8866 (6-10).

The properties of IRE1 as a pro-survival signaling mediator are particularly evident in cancer biology as tumors utilize a hyper-adaptive UPR to overcome physiological and pharmacological stresses. This makes IRE1 inhibition an attractive therapeutic option in oncology, which has been successfully demonstrated in preclinical studies targeting multiple myeloma (6-9), prostate cancer (12), acute myeloid leukemia $(13,14)$ and triple negative breast cancer (TNBC) $(15,16)$. Collectively, these results demonstrate that IRE1 inhibition may be able to address several clinical scenarios in oncology as it may be used as monotherapy or as adjuvant therapy alongside established regimes. An additional solid cancer that IRE1 inhibition may prove to be effective against is Glioblastoma (GB, the most common primary tumor of the Central Nervous System (CNS)). GB is an aggressive, highly recurrent and highly heterogenous cancer. Most patients succumb to the disease despite a comprehensive therapeutic standard of care (SOC) protocol comprising maximal safe resection of the tumor followed by a combination of irradiation and chemotherapy with 
the alkylating agent temozolomide (STUPP protocol) (17). To cope with an hostile microenvironment GB cells need to maintain high metabolic rates and consequently protein synthesis demands (18). This protein demand is met with the help of the UPR, which is inextricably linked to GB pathophysiology (19). Indeed, genetic ablation of IRE1 in GB preclinical models was shown to give rise to smaller and less aggressive tumors $(20,21)$. Moreover by applying an IRE1 activity signature to GB patient cohorts we correlated high levels of IRE1/XBP1s activity with a worse prognosis (22).

To test whether IRE1 RNase pharmacological inhibition could be effective against GB development, we established a novel preclinical murine GB surgical model that fully recapitulates the SOC given to human patients (23). We showed that although SOC and MKC8866 co-administration was beneficial in terms of mouse survival compared to SOC alone, the effect was relatively modest, most likely due to the limited bioavailability of the compound. This points to an urgent need for an IRE1 inhibitor that can cross the BBB to treat GB. In this study we identify and characterize a novel, BBBpermeable IRE1 inhibitor that enhance the efficacy of the $S O C$ and exhibit minimal side-effects in an in vivo surgical model of GB. This IRE1 inhibitor sensitizes GB models to existing therapies, have a profound effect on IRE1 activity in vitro and in cellbased models, and when administered together with temozolomide in vivo, prevents tumor relapse. 


\section{Materials and Methods}

Materials - IRE1 wild-type recombinant protein encoding the cytoplasmic domain (amino acids 465-977) with N-terminal polyhistidine-tag and GST tag was from Sinobiological (Sino Biological Europe GmbH, Eschborn, Germany, \#11905-H20B). The fluorescent probe used for the in vitro IRE1 RNase assay was from Eurogentec (Cy3-CAUGUCCGCAGCGCAUG -BHQ3). Tunicamycin was purchased from Calbiochem (Merck KGaA, Darmstadt, Germany). All the inhibitors were synthesized by and purchased from Enamine (Riga, Latvia).

In silico drug analyses - Docking - IRE1 protein structure (PDB IDs 3P23 \& 4U6R) and libraries of compounds were prepared using Maestro Suites 2015-2018 (Schrödinger Release 2018-4: Schrödinger, LLC, New York, NY, 2018) and specifically utilizing tools within this program such as LigPrep, Glide Dock, Pharmacophore Hypothesis Generation, Glide grid generation and Protein preparation. Structural exploration and peptides preparation were carried out using software Supermimic (24) and MOE (MOE 2018.01: Chemical computing group, Montreal Canada), respectively. UCSF Chimera (25) was used for image generation developed by the Resource for Biocomputing, Visualization, and Informatics at the University of California, San Francisco, with support from NIH P41-GM103311. Hypotheses were tested against ZINC15 and FDA databases using the Supercomputer cluster Hebbe at the C3SE supercomputing centre (Sweden). Potential ADME properties were investigated using QikProp from Maestro Suites 2015-2018 (Schrödinger Release 2018-4: QikProp, Schrödinger, LLC, New York, NY, 2018) as well as swissADME (26). Boiled egg model -Ligand Central Nervous System parameters and boiled-egg diagrams were calculated using SwissADME, $(26,27)$ a comprehensive and free-to-use web portal developed and maintained by the Molecular Modeling Group of the Swiss Institute of Bioinformatics (SIB).

Microscale thermophoresis - The direct binding of the Z4 and Z4P compounds to IRE1 protein was measured using MicroScale Thermophoresis (MST). IRE recombinant protein was labelled using RED-Tris-NTA fluorescent dye (RED-Tris-NTA $2^{\text {nd }}$ Generation, NanoTemper, Munich, Germany; \# MO-L018). For the labelling step, $100 \mu \mathrm{L}$ of $20 \mathrm{nM}$ protein solution is mixed with $100 \mu \mathrm{L}$ of $10 \mathrm{nM}$ RED-Tris-NTA dye in PBST buffer (PBS with $0.05 \%$ Tween-20) and incubated for $30 \mathrm{~min}$ at RT. The protein- 
dye mixture was centrifuged for $10 \mathrm{~min}$ at $4^{\circ} \mathrm{C}$ and $15000 \mathrm{xg}$. For measurement of direct binding, the compounds were analyzed in a 16-point dilution series mixed in a 1:1 ratio with the labelled protein in PBST buffer. The assay was performed in standard Monolith NT.115 Capillaries (NanoTemper; \#MO-K022), and all measurements were performed at $60 \%$ MST power and $60 \%$ excitation power using the Monolith NT.115 Pico machine (NanoTemper). The dissociation constant (Kd) was calculated by taking the average of triplicate normalized fluorescence data using NANOTEMPER analysis software (MO.Affinity Analysis v2.3). The normalized values were converted to fraction-bound data, and the resulting binding curves are plotted using GRAPHPAD PRISM software (GraphPad Software).

IRE1-mediated in vitro RNase assay - This was performed as previously described (28). Briefly, organic molecules were diluted in minimal volume of DMSO and subsequently re-diluted in reaction buffer (20 mM HEPES pH 7.5; $1 \mathrm{mM} \mathrm{MgOAc} ; 50$ $\mathrm{mM} \mathrm{KOAc}$ ). Maximum volume of DMSO per reaction never exceeded $1 \%$. Reaction volume was $25 \mu \mathrm{L}$. Recombinant IRE1 $(0.6 \mu \mathrm{g} /$ reaction) was incubated at room temperature for 10 minutes with varying concentrations (0-100 $\mu \mathrm{M})$ of inhibitor and reaction buffer. The assay relied on the use of fluorescence resonance energy transfer (FRET)—quenched mini Xbp1 RNA substrate probe, which when cleaved by IRE1 emits fluorescence at $590 \mathrm{~nm}$ (cy3) wavelength (28). Subsequently equal volume of mixture of reaction buffer, $20 \mathrm{mM}$ ATP, $2 \mathrm{mM}$ DTT and $1 \mu \mathrm{g}$ of fluorescent probe were added to each sample and fluorescence was read in 96 well plates flat bottom, black polystyrene, matrix active group High Bind (Corning®) every minute for 25 minutes, at $37^{\circ} \mathrm{C}$, using a Tecan 200 plate reader.

Cell culture and treatments - Human glioblastoma U87MG (U87; ATCC) and U251MG (U251; Sigma-Aldrich) cell lines were authenticated as recommended by AACR (http://aacrjournals.org/content/cell-line-authentication-information) and regularly tested for the absence of mycoplasma using MycoAlert ${ }^{\circledR}$ (Lonza, Basel, Switzerland) or MycoFluor (Invitrogen, Carlsbad, CA, USA). U87 and U251 were grown in DMEM Glutamax (Invitrogen, Carlsbad, CA, USA) supplemented with 10\% FBS. GB primary RADH87 (29) cells were grown in DMEM supplemented with $10 \%$ FBS in a $5 \% \mathrm{CO} 2$ humidified atmosphere at $37^{\circ} \mathrm{C}$. GB cell lines were modified for IRE1 activity by overexpressing dominant negative (DN) forms of IRE1 that lack the RNase domain 
(IRE1.NCK or IRE1 Q780stop) as previously described (22, 30). For XBP1s induction experiments, tunicamycin was used at $1 \mu \mathrm{g} / \mathrm{mL}$ for the indicated periods of time. For inhibitor cell toxicity assays cells were plated in 96 well plates at 5000 cells per well and treated with $0,5,10,25,50,100,250,500,1000$ and $2500 \mu \mathrm{M}$ concentrations of each inhibitor. After 6 days of incubation, WST1 reagent (Roche) was added to each well and post 2-hour incubation the plate was read using a Tecan 200 colorimeter. For TMZ sensitivity assays cells were plated in a 96 well plate at 5000 cells per well and co-treated with $0,5,10,25,50,100,250,500,1000$ and $2500 \mu \mathrm{M}$ of TMZ plus a nontoxic dose of inhibitor. After 6 days of incubation, WST1 reagent (Roche) was added to each well and post 2-hour incubation the plate was read using a Tecan 200 colorimeter.

Western blotting - All IRE1 signaling analyses were carried out as described previously (31). Cells grown on 6 well plates were washed with PBS and lysed with RIPA lysis buffer at $4^{\circ} \mathrm{C}$, over 25 minutes to extract protein. IRE1 and phosphorylated IRE1 were stained using anti-IRE1 antibody (Anti-human; rabbit polyclonal; SantaCruz Biotechnologies, $\mathrm{H}-190$ ) and pS724-IRE1 antibody (Anti-human; rabbit polyclonal; Abcam, ab48187), respectively. The phosphorylated form of elF2 $\alpha$ was stained with anti-phospho-elF2 $\alpha$ (Ser51) Antibody \#9721 (CellSignalling ${ }^{\circledR}$ ). Actin was used as a loading control ( $\beta$-Actin (C4): sc-47778; SantaCruz Biotechnologies). Cell extracts were resolved by SDS-PAGE and transferred to nitrocellulose membranes for 30 minutes using a Trans-Blot ${ }^{\circledR}$ Turbo $^{\mathrm{TM}}$ (BioRad $®$ Transfer System \#1704150). The resulting membranes were incubated with primary antibodies for 16 hours at $4^{\circ} \mathrm{C}$, washed with PBST, and incubated for 1 hour with goat anti-rabbit or goat anti-mouse secondary antibodies at room temperature (Invitrogen, Carlsbad, CA, USA) prior revelation using chemiluminescence (ECL RevelBIOt ${ }^{\circledR}$ Intense, Ozyme).

Proteomic analyses - Untreated and MKC8866 and Z4-treated (during 48 hours) parental U251 and RADH87 GB cells as well as DN IRE1 U251 and RADH87 cells were lysed with lysis buffer composed of $20 \mathrm{mM}$ Tris $\mathrm{pH} \mathrm{8,} 1.5 \mathrm{mM}$ EDTA, $150 \mathrm{mM}$ $\mathrm{NaCl}, 1 \%$ Triton $\mathrm{X}-100,0.1 \%$ SDS, supplemented with proteases and phosphatases inhibitor cocktails (Roche). Total proteins were precipitated with $80 \%$ ice-cold acetone. Washed pellet were then denatured with $8 \mathrm{M}$ urea in Tris- $\mathrm{HCl} 0.1 \mathrm{mM}$, reduced with 5 $\mathrm{mM}$ TCEP for 30 minutes, and then alkylated with $10 \mathrm{mM}$ iodoacetamide for 30 minutes 
in the dark. Double digestion was performed with endoproteinase Lys-C (Ref 12505061, Wako) at a ratio $1 / 100$ (enzyme/proteins) in $8 \mathrm{M}$ urea for $4 \mathrm{~h}$, followed by an overnight modified trypsin digestion (Ref V511A, Promega) at a ratio 1/100 (enzyme/proteins) in $2 \mathrm{M}$ urea. Both Lys-C and Trypsin digestions were performed at $37^{\circ} \mathrm{C}$. Peptide mixtures were then desalted on $\mathrm{C} 18$ spin-column and dried on SpeedVacuum before LC-MS/MS analysis. Samples were analyzed using an Ultimate 3000 nano-RSLC (Thermo Scientific, San Jose California) coupled in line with a LTQOrbitrap ELITE mass spectrometer via a nano-electrospray ionization source (Thermo Scientific, San Jose California). Proteins were identified and quantified by database searching using SequestHT (Thermo Fisher Scientific) with Proteome Discoverer 2.4 software (PD2.4, Thermo Fisher Scientific) against Homo sapiens reviewed SwissProt database. Peptides and proteins were filtered with a false discovery rate (FDR) at $1 \%$ and their quantification was based on XIC (Extracted lon Chromatogram). Raw data, Proteome Discoverer parameters and results are provided at PRIDE protein identification database. Data Availability - The mass spectrometry proteomics data have been deposited to the ProteomeXchange Consortium via the PRIDE partner repository with the dataset identifier PXD026908". The dataset is available at: http://www.ebi.ac.uk/pride.

Proteomic Data analyses and representation - Differential expression analysis was executed to identify the impact of Z4 treatment and IRE1 knock on the proteome, comparing the parental strains of U251 and RADH7 cell lines with the Z4-treated, dimethyl sulfoxide (DMSO)-treated and DN forms of them. The statistical analysis was based on the non-parametric method of rank products, using the RankProd (R/Bioconductor) package (32). Its RP and topGene functions were utilized to identify the most significantly over and under-expressed proteins, setting the $p$-value threshold at 0.05 . Differentially expressed proteins in the wild type - Z4-treatement comparison with absolute log fold change value lower than that of wild type - DMSO comparison, were filtered out, as their regulation cannot be exclusively attributed to the effect of Z4 administration. BiolnfoMiner $(33,34)$ was used for the functional interpretation of the sets of significantly perturbed proteins and the comparative analysis of the derived semantic networks. Pathway analysis was performed, exploiting the annotation of Gene Ontology (Biological Process domain - GO-BP) (35), with hyper-geometric and adjusted $p$-value thresholds at 0.05 . A network of statistically important GO-BP terms 
was identified for each list of proteins. Then, all significantly over-represented terms were clustered, using the Resnik semantic similarity measure (36) In each iteration of the agglomerative clustering process, the most similar pair of terms was substituted with its most informative common ancestor (mica). Semantic similarity threshold was set to 0.25 , as lower values might produce semantic clusters, with overly generic semantic description. Examining the membership of each GO-BP cluster and the initial lists of over-represented terms, a binary association matrix was constructed, to reveal and compare the membership profiles of the regulated sets of proteins in cellular functionality.

Tumor cell orthotopic implantation - Tumor cells (U87-Luc (37)) were implanted into the brain of immunodeficient NMRI-Foxn1nu/Foxn1nu, 8 weeks old male mice (Janvier Laboratories, Laval, France). All animal procedures met the European Community Directive guidelines (Agreement B35-238-40 Biosit Rennes, France/ No DIR 13480) and were approved by the local (University of Rennes) ethics committee and ensuring the breeding and the daily monitoring of the animals in the best conditions of wellbeing according to the law and the rule of 3R (Reduce-Refine-Replace). U87-Luc cells were implanted in the mouse brain by intracerebral injection followed by tumor growth monitoring using bioluminescence (PhotonIMAGER ${ }^{\text {TM }}$ systems, BIOSPACE LAB). The mice were anesthetized intraperitoneally (i.p. mix Xylazine $10 \mathrm{mg} / \mathrm{kg}$ and Ketamin 60 $\mathrm{mg} / \mathrm{kg}$ ) and then fixed on a stereotactic frame. This framework makes it possible to manipulate the brains of living animals, and to reach isolated areas of the brain precisely relative to markings visible to the naked eye through the use of threedimensional coordinates. After incising the scalp, the stereotaxic coordinates were calculated for injection of tumor cells into a specific point of the brain, and reproducible for all the mice used. In the study, the tumor cells $\left(5 \times 10^{4}\right.$ cells per mice in $\left.1 \mu \mathrm{L}\right)$ were injected at bregma $0,2.2 \mathrm{~mm}$ to the left of the bregma and $3.2 \mathrm{~mm}$ deep to perform the implantation at the level of the striatum.

Mouse treatments - Four days after tumor cells implantation, Z4P treatments were started consisting in treatments of $300 \mu \mathrm{g} / \mathrm{kg} /$ day intraperitonially. For the RNA quantification experiment, performed on tumors generated in mice, three mice per group were treated according to the methods above. Mice were sacrificed at day 34 and the brains were removed and extract the tumor. The samples were used to extract 
RNA for the analysis, as described below. For the survival experiment, performed on tumors generated in mice, co-treatment with TMZ was performed one week after the beginning of $Z 4$ treatment ( $n=7$ per group for the TMZ and TMZ+Z4P (combo), $n=8$ per group for control and Z4P). The animals were injected with $10 \mathrm{mg} / \mathrm{kg} /$ day of TMZ delivered intraperitoneally for ten days. All the treatments were performed with 2 days of rest each week. Survival was measured as the time between implantation and sacrifice, which was performed by cervical dislocation in case of critical clinical signs or loss of weight $>10 \%$.

Bioluminescence monitoring - Mice were evaluated with in vivo bioluminescence imaging every 3 days starting from the day 4 , for the first week, and weekly onward for tumor progression and followed for signs of neurologic deterioration daily. Mice were injected i.p. with $100 \mu \mathrm{L}$ of luciferin (Promega, Charbonnières-les-Bains, France). The luciferin was allowed to circulate for 5 min before the mice were anesthetized with a mix of $\mathrm{O}_{2}$ and isoflurane $(2.5 \%)$.

Quantitative real-time PCR - Total RNA was prepared using TRIzol reagent (Invitrogen, Carlsbad, CA, USA). All RNAs were reverse-transcribed with Maxima Reverse Transcriptase (Thermo Scientific, Waltham, MA, USA), according to manufacturer protocol. qPCR was performed via a StepOnePlus ${ }^{\mathrm{TM}}$ Real-Time PCR Systems from Applied Biosystems and the SYBR Green PCR Core reagents kit (Takara). Analysis was carried out using QuantStudio ${ }^{\mathrm{TM}}$ Design and Analysis software version 1.3.1. Three technical repeats were performed per experiment and at least three biological repeats were performed per point per experiment. Each sample was extract individually for RNA and performed the quantification of mRNA levels by qPCR for several targets. These data were then plotted using Morpheus' tool from the Broad institute (https://software.broadinstitute.org/morpheus). The primers used in the qPCR analyses are presented in Table $\mathbf{S 1}$

Statistical analyses - Data are presented as mean \pm SEM or \pm SD as indicated in each figure. Statistical significance ( $P<0.05$ or less) was determined using unpaired $t$-tests or ANOVA as appropriate and performed using GraphPad Prism software (GraphPad Software, San Diego, CA, USA). Curve's extrapolations were performed 
using curve fit hypotheses by GraphPad Prism software (GraphPad Software, San Diego, CA, USA).

Chemical syntheses - General information - Commercially available reagents and solvents were purchased from Enamine, Fluorochem, Sigma-Aldrich, Alfa Aesar, Acros and were used as received. All reactions under inert atmosphere were performed under an argon atmosphere. Petroleum ether (PE) refers to petroleum ether with $40-60^{\circ} \mathrm{C}$. Thin-layer chromatography (TLC) was performed on TLC silica gel 60 F254 aluminum plates. Compounds were visualized by exposure to UV light or by dipping the plates into potassium permanganate or phosphomolybdic acid solution followed by heating. Manual flash column chromatography was carried out using silica gel (particle size 40-63 $\mu \mathrm{m}$ ) with step gradient elution as indicated. Automated flash column chromatography was carried out on an Interchim PuriFlash 450 using FlashPure Ecoflex silica cartridges (irregular particle size $50 \mu \mathrm{m}$ ) with NMR spectra were acquired on $300 \mathrm{MHz}$ spectrometers and were referenced to the residual solvent. All chemical shifts are reported in parts per million (ppm). Abbreviations used in the description of resonances are $s$ (singlet), $d$ (doublet), $t$ (triplet), $q$ (quartet), sept (septet), a (apparent), bs (broad singlet), and $\mathrm{m}$ (multiplet). Coupling constants $(\mathrm{J})$ are quoted to the nearest $0.1 \mathrm{~Hz}$. HPLC-MS analyses were performed on a Shimadzu Prominence system couple to an Advion ESI mass spectrometer using a Thermoscientific Hypersil Gold $\mathrm{aQ}$ column chromatography $(5 \mu, 250 \times 4.6 \mathrm{~mm})$ [method: binary gradient, solvent $A=\mathrm{H} 2 \mathrm{O}+0.1 \%$ TFA, solvent $B=\mathrm{MeCN}+0.1 \%$ TFA, flow $=1 \mathrm{~mL} / \mathrm{min}, 20$ to $100 \% \mathrm{~A} / \mathrm{B}]$. Racemic Z4P synthesis - 4- $(4-$ methoxyphenyl)butan-2-amine - To a $250 \mathrm{~mL}$ RB flask filled with methanol (150 mL) was added 4-(4-methoxyphenyl)butan-2-one ( $5 \mathrm{~g}, 28.05 \mathrm{mmol}, 1$ eq.) and ammonium acetate $(12.97 \mathrm{~g}, 168.3 \mathrm{mmol}, 6 \mathrm{eq}$.) and the reaction mixture was left to stir at room temperature for $30 \mathrm{~min}$. Temperature is then lowered to $0^{\circ} \mathrm{C}$ with an ice bath and sodium cyanoborohydride ( $2.64 \mathrm{~g}, 42.07 \mathrm{mmol}, 1.5 \mathrm{eq}$.) was added portion wise. The ice batch was then removed and the reaction left to stir overnight at room temperature. TLC monitoring indicated full consumption of the starting material and the reaction was subsequently quenched at $0^{\circ} \mathrm{C}$ by addition of $\mathrm{HCl} 1 \mathrm{M}(200 \mathrm{~mL})$. After $15 \mathrm{~min}$ of stirring, the reaction mixture was concentrated by rotary evaporation to remove most of the methanol and then extracted with diethyl ether $(2 \times 100 \mathrm{~mL})$. The aqueous layer is isolated and basified by addition of concentrated $\mathrm{NaOH}$ until $\mathrm{pH} \geq 10$, and then sodium 
chloride is added until saturation, followed by extraction with DCM $(3 \times 100 \mathrm{~mL})$. The combined organic layers were dried over anh. $\mathrm{MgSO}_{4}$, filtered, evaporated to dryness by rotary-evaporation and the residue dried under high-vacuum to yield $4.2 \mathrm{~g}$ of crude ( $83 \%$ yield, 80 to $85 \%$ yield on average). Crude product was pure enough to be used without further purification, but if needed, purification can be performed by silica gel column chromatography with gradient elution (100:0-90:10 DCM/MeOH).<smiles>COc1ccc(CCC(C)N)cc1</smiles>

${ }^{1} \mathrm{H}$ NMR (300.16 MHz, $\left.\mathrm{CDCl}_{3}, 20^{\circ} \mathrm{C}\right): \delta=7.10(\mathrm{dt}, 2 \mathrm{H}, \mathrm{CH} 7,8) ; 6.82(\mathrm{dt}, 2 \mathrm{H}, \mathrm{CH} 9,10)$; 3.78 (s, 3H, $\mathrm{CH}_{3}$ 12); 2.90 (h, 1H, CH2); 2.60 (qdd, 2H, $\mathrm{CH}_{2}$ 5); 1.62 (m, 2H, $\mathrm{CH}_{2}$ 4); $1.10\left(\mathrm{~d}, 3 \mathrm{H}, \mathrm{CH}_{3}\right.$ 1). 4-nitrophenyl (2,5-dimethylphenyl)carbamate - To a $250 \mathrm{~mL} \mathrm{RB}$ flask filled with dry AcOEt $(100 \mathrm{~mL})$ and under argon atmosphere was added successively the 4-nitrophenyl chloroformate $(2.0 \mathrm{~g}, 9.9 \mathrm{mmol}, 1.1 \mathrm{eq}$.) potassium carbonate $(1.37 \mathrm{~g}, 9.9 \mathrm{mmol}, 1.1 \mathrm{eq}$.) and 2,5-dimethylaniline $(1.12 \mathrm{~mL}, 9.0 \mathrm{mmol}, 1$ eq.). The reaction is left stirring at room temperature and followed by TLC. After $2 \mathrm{~h}$, the reaction mixture is diluted with AcOEt $(100 \mathrm{~mL})$, transferred in a separatory funnel and washed successively with $1 \mathrm{M}$ citric acid $(100 \mathrm{~mL})$, sat. sodium carbonate (100 $\mathrm{mL}$ ), and brine $(100 \mathrm{~mL})$. The organic layer is dried over anh. $\mathrm{MgSO}_{4}$, filtered, evaporated to dryness by rotary-evaporation and the residue dried under high-vacuum to afford $1.9 \mathrm{~g}$ of (2,5-dimethylphenyl)carbamate ( $74 \%$ yield) as a pale beige solid, used without further purification.<smiles>O=C(Nc1cc(I)ccc1I)Oc1ccc([N+](=O)[O-])cc1</smiles>

${ }^{1} \mathrm{H} \mathrm{NMR}\left(300.16 \mathrm{MHz}, \mathrm{CDCl}_{3}, 20^{\circ} \mathrm{C}\right): \delta=8.29$ (dt, $\left.2 \mathrm{H}, \mathrm{CH} 14,15\right) ; 7.64$ (bs, $1 \mathrm{H}, \mathrm{NH}$ ); 7.41 (dt, $2 \mathrm{H}, \mathrm{CH}$ 12,13); 7.11 (d, 1H, CH3); 6.93 (d, 1H, CH 4); 6.78 (bs, $1 \mathrm{H}, \mathrm{CH}$ ); $2.32\left(\mathrm{~d}, 6 \mathrm{H}, \mathrm{CH}_{3}\right.$ 1,6). 1-(2,5-dimethylphenyl)-3-(4-(4-methoxyphenyl)butan-2-yl)urea - To a $25 \mathrm{~mL}$ RB flask filled with dry DCM (12 mL) and under argon atmosphere was added 4-nitrophenyl (2,5-dimethylphenyl)carbamate (639 mg, $2.231 \mathrm{mmol}, 1$ eq.) and triethylamine ( $311 \mu \mathrm{L}, 2.231 \mathrm{mmol}, 1$ eq.), followed by the 4-(4-methoxyphenyl)butan- 
2-amine (400 mg, $2.231 \mathrm{mmol}, 1$ eq.). The reaction was left stirring at room temperature and TLC monitoring showed full conversion after $1 \mathrm{~h}$. The reaction mixture was then concentrated to dryness by rotary-evaporation and the solid residue triturated and sonicated in diisopropyl ether $(10 \mathrm{~mL})$, then filtered. The solid was collected and resuspended in cold water $(10 \mathrm{~mL})$ to be sonicated, followed by filtration (repeat twice). Finally, the solid was collected and dried under high-vacuum to afford $564 \mathrm{mg}$ of 1 (2,5-dimethylphenyl)-3-(4-(4-methoxyphenyl)butan-2-yl)urea (77\% yield) as a pale white solid, used without further purification.

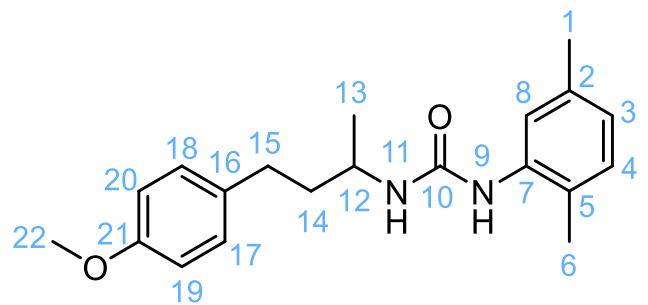

${ }^{1} \mathrm{H}$ NMR $\left(300.16 \mathrm{MHz},\left(\mathrm{CD}_{3}\right)_{2} \mathrm{SO}, 20^{\circ} \mathrm{C}\right): \delta=7.81$ (s, $1 \mathrm{H}, \mathrm{NH}$ ); 7.67 (s, $1 \mathrm{H}, \mathrm{CH}$ ); 7.12 (dt, 2H, CH 17,18); 6.95 (d, 2H, CH 3,4); 6.83 (dt, 2H, CH 19, 20); 6.64 (d, $1 \mathrm{H}$, $\mathrm{NH} 11) ; 3.70\left(\mathrm{~s}, 3 \mathrm{H}, \mathrm{CH}_{3} 22\right) ; 3.55(\mathrm{~m}, 1 \mathrm{H}, \mathrm{CH} 12) ; 2.56(\mathrm{~m}, 2 \mathrm{H}, \mathrm{CH} 215) ; 2.20$ (s, 3H, $\mathrm{CH}_{3}$ 1); 2.14 (s, 3H, $\mathrm{CH}_{3}$ 6); 1.64 (sept, $2 \mathrm{H}, \mathrm{CH}_{2}$ 14); 1.08 (d, 3H, $\mathrm{CH}_{3}$ 13). 1-(2,5dimethylphenyl)-3-(4-(4-hydroxyphenyl)butan-2-yl)urea (Z4P) - To a $100 \mathrm{~mL}$ RB flask filled with dry DCM $(30 \mathrm{~mL})$, under argon atmosphere was added 1-(2,5dimethylphenyl)-3-(4-(4-methoxyphenyl)butan-2-yl)urea (300 mg, $0.92 \mathrm{mmol}, 1$ eq.). After cooling to $0^{\circ} \mathrm{C}$, boron tribromide ( $1 \mathrm{M}$ in $\mathrm{DCM}, 4.6 \mathrm{~mL}, 5$ eq.) was added slowly over the course of a few minutes. The reaction mixture was left stirring for $1 \mathrm{~h}$ at $0^{\circ} \mathrm{C}$, and then warmed to room temperature and left stirring overnight. TLC monitoring showed full consumption of the starting material and the reaction was quenched by addition of ice $(20 \mathrm{~g})$ and stirred for 30 minutes before being transferred to a separatory funnel. Layers were separated and the aqueous layer extracted with DCM $(2 \times 40 \mathrm{~mL})$ and AcOEt $(2 \times 40 \mathrm{~mL})$. The combined organic layers were dried over anh. $\mathrm{MgSO}_{4}$, filtered, evaporated to dryness by rotary-evaporation and the residue dried under highvacuum to yield $258 \mathrm{mg}$ of crude. $50 \mathrm{mg}$ of crude product was purified by silica gel column chromatography (3g Silica, ratio 1:60) with solid loading and isocratic elution (75:25 EP/Acetone) to afford $47 \mathrm{mg}$ of 1-(2,5-dimethylphenyl)-3-(4-(4hydroxyphenyl)butan-2-yl)urea (Z4P) as a white powder. 


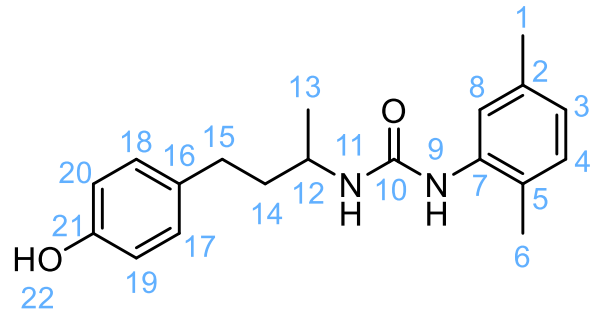

${ }^{1} \mathrm{H}$ NMR $\left(300.16 \mathrm{MHz},\left(\mathrm{CD}_{3}\right)_{2} \mathrm{CO}, 20^{\circ} \mathrm{C}\right): \delta=8.10$ (bs, $\left.1 \mathrm{H}, \mathrm{OH} 22\right) ; 7.80$ (s, $1 \mathrm{H}, \mathrm{NH}$ ); 7.09 (s, 1H, CH8); 7.03 (dt, 2H, CH 17,18); 6.97 (d, 1H, CH4); 6.74 (dt, 2H, CH 19,20); 6.69 (d, 1H, CH3); 5.92 (d, 1H, NH 11); 3.87 (sept, 1H, CH 12); 2.60 (m, 2H, CH2 15); 2.24 (s, 3H, $\mathrm{CH}_{3}$ 1); 2.15 (s, 3H, $\left.\mathrm{CH}_{3} 6\right) ; 1.71$ (m, 2H, $\left.\mathrm{CH}_{2} 14\right) ; 1.16$ (d, 3H, $\mathrm{CH}_{3} 13$ ). ${ }^{13} \mathrm{C}$ NMR $\left(75.47 \mathrm{MHz},\left(\mathrm{CD}_{3}\right)_{2} \mathrm{CO}, 20^{\circ} \mathrm{C}\right): \delta=156.3$ (C 21); 155.9 (CO 10); 139.2 (C 16); 136.3 (C 2); 133.8 (C 7); $130.7(\mathrm{CH} 4) ; 130.1(\mathrm{CH} 17,18) ; 124.8(C 5) ; 123.7(\mathrm{CH}$ 3); 122.5 (C 7); 115.92 (CH 19,20); $46.0(\mathrm{CH} 12) ; 40.4\left(\mathrm{CH}_{2} 14\right) ; 32.3\left(\mathrm{CH}_{2} 15\right) ; 21.8$ $\left(\mathrm{CH}_{3} 13\right) ; 21.3\left(\mathrm{CH}_{3} 1\right) ; 17.7\left(\mathrm{CH}_{3} 6\right)$. MS (ESI+): $\mathrm{m} / z(\%) 313.2(100)\left[\mathrm{M}_{+} \mathrm{H}^{+}\right]$. Chiral HPLC separation - The preparative chiral HPLC separation was performed on an Agilent 1260 Infinity unit (pump G1311C, autosampler G1329B, DAD G1365D and fraction collector G1364C), and monitored by Agilent OpenLAB CDS Chemstation LC. Samples were dissolved in a mixture of ethanol and hexane (50/50) and eluted on a Chiralpak ID $(250 \times 10 \mathrm{~mm})$ column, with hexane / ethanol / dichloromethane $(80 / 10 / 10)$ as mobile phase, flow-rate $=5 \mathrm{~mL} / \mathrm{min}$, UV detection at $254 \mathrm{~nm}$, yielding two enantiomers $\left(R T_{A}=7.28 \mathrm{~min}\right.$ and $\left.R T_{B}=9.12 \mathrm{~min}\right)$ with ee> $99.5 \%$. Optical rotary power measurements - Absolute stereochemistry of enantiomers $A$ and $B$ were determined by measuring their optical rotary power and compared with a Z4P enantiomer of known stereochemistry, Z4P-R (in-house synthesis). Measurements were performed on Perkin Elmer polarimeter 341 with sodium lamp (589 $\mathrm{nm}$ ) and 1$\mathrm{dm}$ path at $20^{\circ} \mathrm{C}$ using $0.5 \mathrm{mg} / \mathrm{mL}$ solutions of enantiomer $\mathrm{A}$, enantiomer $\mathrm{B}$ and Z4P. $R$ in acetonitrile. Results are the average of 3 measurements (1s integration time). Enantiomer A: average optical rotation $-0.023^{\circ},[\alpha]_{D}=-46$; Enantiomer B: average optical rotation $+0.021^{\circ},[\alpha]_{\mathrm{D}}=+42 ;$ Z4P- $R$ : average optical rotation $+0.023^{\circ},[\alpha]_{\mathrm{D}}=+$ 46. Enantiomer $\mathrm{A}=\mathrm{Z} 4 \mathrm{P}-\mathrm{S}$ \& Enantiomer $\mathrm{B}=\mathrm{Z} 4 \mathrm{P}-\mathrm{R}$. 


\section{Results}

We have previously described a drug discovery pipeline which uses structural analysis of the IRE1 crystal structure to yield groups of FDA approved compounds that can inhibit IRE1 activity in vitro and sensitize cellular models of GB to temozolomide (TMZ) (38). Despite this wealth of new information, the fact remained that these drugs could not cross the BBB, had known clinical toxicity profiles and were not specific binders to IRE1 itself. To solve these issues, we used our drug discovery pipeline to identify novel molecules fulfilling a specific set of criteria: (i) to be able to specifically bind to the IRE1 kinase domain; (ii) to have a low toxicity profile in experimental GB models in vitro and in vivo; (iii) to block IRE1 activity in vitro and in vivo; (iv) to be able to cross the BBB; (v) to sensitize GB experimental models to chemotherapy in vitro and in vivo and; (vi) to be without a known clinical designation.

\section{Identification of novel IRE1 modulator candidates through an in silico} pharmacophore screen - Applying the IRE1 modulator discovery pipeline (38), we screened the ZINC15 (39) database to identify compounds able to bind onto the ATPbinding pocket of the IRE1 kinase domain. For that, we utilized pharmacophore binding hypotheses between the IRE1 kinase domain and fragments of the IRE1 structure itself $(38,40)$ (Figure 1A). We also looked for compounds which were predicted to dock more favorably than its natural substrate, ATP (Table S2). This was done to ensure maximal specificity due to the structural similarity between the kinase and the fragments. The resulting compound library from the pharmacophore ZINC15 screen were then computationally docked in the IRE1 ATP binding pocket using Glide Dock of the Maestro Suite. The candidates with docking scores superior to ATP were chosen to be further tested to validate their ability to modulate IRE1 activity in vitro. The prime candidate, (herein named Z4) was then extensively tested for its ability to modulate IRE1 activity. Once validated in vitro and in cell-based models, the molecule was tested in a pre-clinical model of GB (Figure 1A).

\section{In silico identification of a novel IRE1 modulator candidate capable of crossing} the BBB - At this stage we had identified a compound with the ability to bind onto IRE1 in silico. However, to broaden the repertoire of compounds to test we sought to identify Z4-like compounds. To do this, we took a structural approach by using Z4 as a template to produce a group of structurally similar small molecules. This was done by applying 
a Tanimoto similarity coefficient (41) to the Z4 structure, the results of which were once again screened against the ZINC database.

A

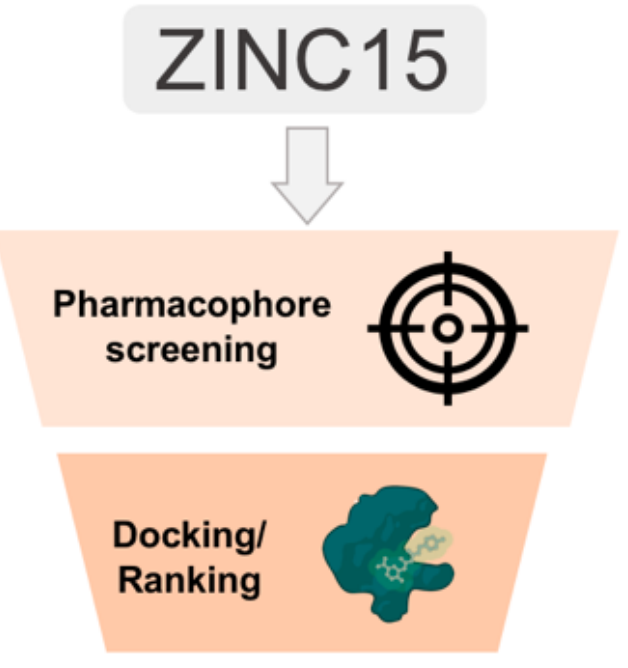

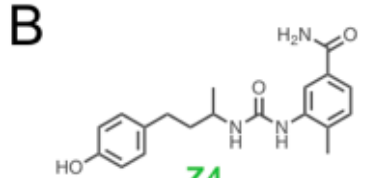

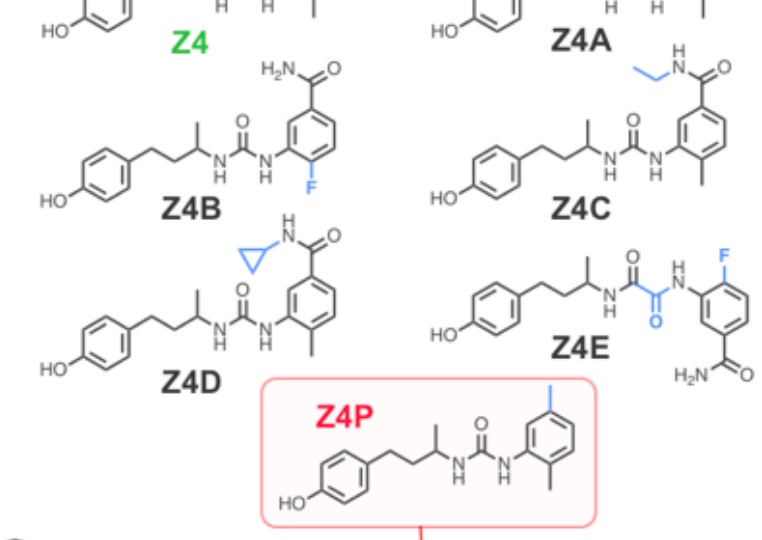

C

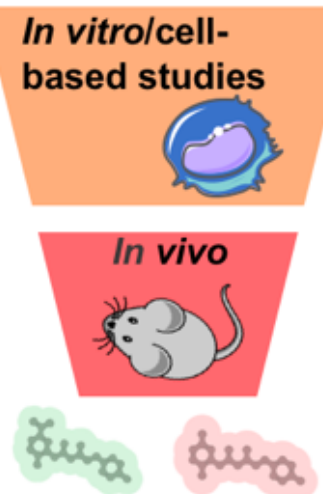

HIT compounds
Z4

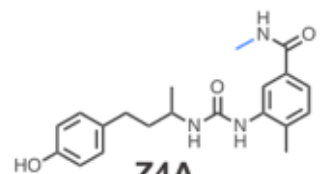

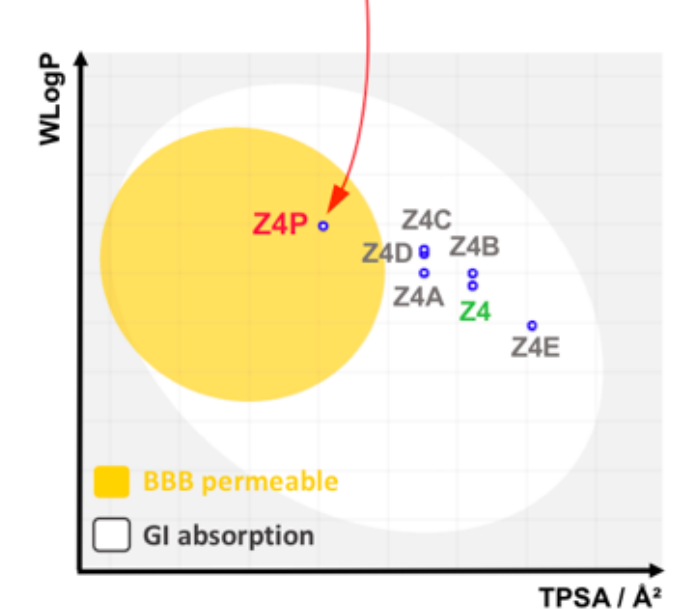

Figure 1: Pipeline of Z4 analogues discovery. (A) The screening based on pharmacophore modelling led to the identification of Z4 as a putative IRE1 inhibitor candidate. The Z4 structure was confronted against the Tanimoto similarity coefficient and screened against the ZINC database to produce the top 100 similar compounds to Z4 with a better or as good as docking score. These compounds were docked into the IRE1 structure as described, and the top 20 binders (using Z4 as the positive control) were chosen for further investigation. These analogues were characterized in vitro and in vivo and the HIT compounds were chosen for the study (B) The bi-dimensional organic structures of Z4A-Z4E and Z4P. (C) The top 20 compounds described in (A) were further assessed for their capability to cross the blood brain barrier, using the egg yolk graphical representation from SwissADME where WLOGP is plotted against TPSA. Compound Z4P was selected for further investigation on top of Z4A-E based on its predicted ability to cross the BBB.

The top 100 similar-to-Z4 hits were then docked to the IRE1 crystal structure to identify compounds with as-good-or-better docking scores than Z4 itself (Figure 1A, Table 1 and Table S2). We then searched the top 20 compounds with superior docking scores to Z4 for candidates predicted to have BBB crossing capabilities as determined using two in silico approaches (QikProp from the Maestro Suite (Schrödinger Release20184: Schrödinger, LLC, New York, NY, 2018) and the online tool swissADME (26)). 
These allowed us to identify properties that may be used as predictors of the likelihood for a molecule to cross the BBB, such as lipophilicity, molecular weight, total polar surface, rotatable bonds and hydrogen bond donors. This process identified a single compound (Z4P) that was predicted to cross the BBB (Figure 1B, 1C and Table 1). We also selected additional candidates with docking scores superior to Z4 for further investigation. This was done to compare their effects on IRE1 signaling in vitro and as a retrospective cross-validation control to show that our in silico pipeline could robustly select novel molecules that can modulate IRE1 activity in silico, in vitro and in vivo. This process led to the identification of 6 compounds (Z4A-Z4E \& Z4P; Figure 1B and C). Z4 family members physicochemical properties important for BBB permeation are presented in Table 1 and their molecular weights and docking score to the IRE1 kinase domain are presented in Table 2.

\begin{tabular}{lcccc}
\hline ADME Property & Log Po/w & $\begin{array}{c}\text { Hydrogen } \\
\text { Bond } \\
\text { Donors }\end{array}$ & $\begin{array}{c}\text { Topological } \\
\text { Polar } \\
\text { Surface } \\
\text { Area }\left(\AA^{2}\right)\end{array}$ & $\begin{array}{c}\text { Rotatable } \\
\text { bonds }\end{array}$ \\
\hline $\begin{array}{l}\text { Median values - } \\
\text { marketed CNS }\end{array}$ & $\leq 3$ & $<3.5$ & $40<$ TPSA $<90$ & $<8$ \\
drugs23,29,30 & & & & \\
Z4 QikProp & 1.767 & 5 & 120.48 & 7 \\
Z4 swissADME & 2.57 & 4 & 104.45 & 8 \\
Z4A QikProp & 2.89 & 4 & 105.25 & 7 \\
Z4A swissADME & 2.91 & 4 & 90.46 & 9 \\
Z4B QikProp & 1.79 & 5 & 120.48 & 7 \\
Z4B swissADME & 2.5 & 4 & 104.45 & 8 \\
Z4C QikProp & 3.32 & 4 & 104.37 & 8 \\
Z4C swissADME & 3.23 & 4 & 90.46 & 10 \\
\hline Z4D QikProp & 3.54 & 4 & 104.14 & 8 \\
Z4D swissADME & 3.30 & 4 & 90.46 & 10 \\
Z4E QikProp & 4.05 & 3 & 66.59 & 6 \\
Z4E swissADME & 2.1 & 4 & 121.52 & 9 \\
Z4P QikProp & 3.80 & 3 & 66.54 & 6 \\
Z4P swissADME & 3.67 & 3 & 61.36 & 7 \\
\hline
\end{tabular}

Table 1: Z4 family members ADME properties. Data representing compounds Z4, Z4A-E and Z4P for predicted ADME properties, Glide Dock results (scores represented are the result of XP (extra precision) docking utilizing Maestro version 2018.11).

\begin{tabular}{lccccccc}
\hline & Z4 & Z4A & Z4B & Z4C & Z4D & Z4E & Z4P \\
\hline Molecular Weight (Da) & 341.40 & 355.43 & 345.37 & 369.46 & 381.47 & 373.38 & 312.41 \\
Docking score & -7.406 & -9.060 & -8.984 & -8.524 & -8.239 & -8.040 & -7.394 \\
\hline
\end{tabular}

Table 2: Z4 family members molecular weights and docking scores.

IRE1-fragment-derived organic molecules dock onto the ATP binding pocket of IRE1 in silico and in vitro - The Z4 family of compounds are small, nitrogen rich para 
substituted phenols that interact with the ATP binding pocket through van der Waals interactions and hydrogen bonding by the phenolic hydroxyl group to Cys645 and hydrogen bonding between polar atoms at the opposite end of the molecules with Asp711 and Asp688 or Lys690 in the phosphate binding region of the pocket (Figure 2A). All dockings were performed using Glide extra precision (XP) mode (42) and the OPLS-2005 force field (43).

A

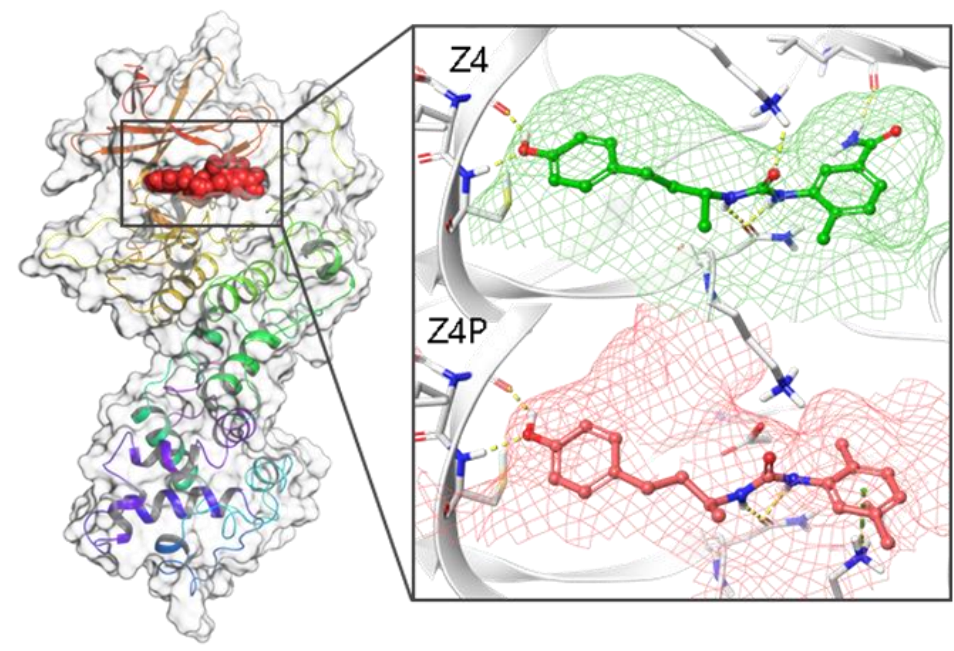

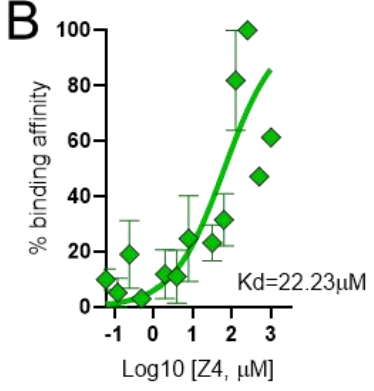

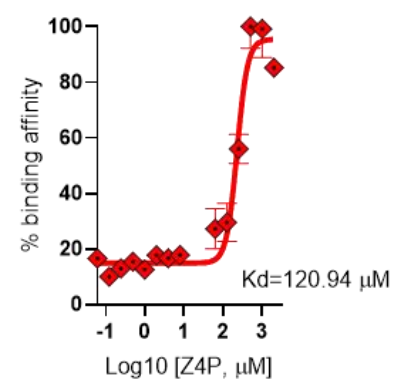

Z4P - R
C

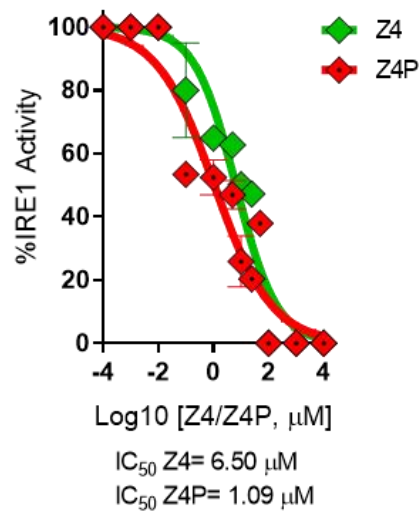

$\mathrm{D}$

Z4P - S

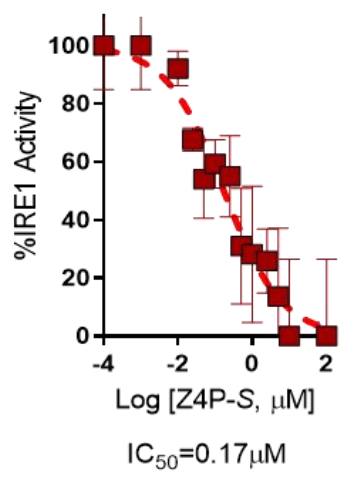

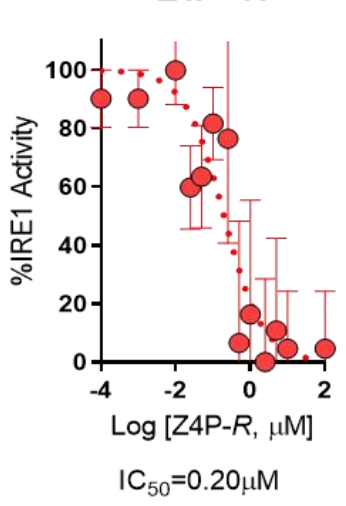

Figure 2: Z4-derived compounds interact with the ATP binding pocket of IRE1 in silico and in vitro. (A) Glide docking poses of Z4 and Z4P in IRE1 ATP-binding pocket (PDB ID 3P23)). (B) Human recombinant IRE1 ( $n=3)$ protein was used as fluorescently labelled target at final concentrations of 20 $n M$ in microscale thermophoresis experiments. Z4 and Z4P were used as ligands in the indicated concentration range. The experimental data were normalized, and fitted to a saturation one-site binding equation. Kd value calculated from the fit are shown in the figure. Activity of IRE1 in the presence of increasing concentrations of Z4, Z4P (C) and its enantiomers Z4P (S) and Z4P (R). (D) from FRET signal given out upon fluorescent probe cleavage over 25 minutes incubation. The $I C_{50}$ calculated from the fitting step are shown in the figure. Symbols and error bars represent mean values $\pm S D$.

Of the different compounds obtained, none was identified as a key binder using the sulfonamide- 'KIRA-like'-bound crystal structure 4U6R, in which ligand-binding induces 
a shift outward of the $\alpha \mathrm{C}$-helix, rupture of the Lys-Glu bridge and dealignment of the R-spine. This slight distortion relative to the ATP bound form has been claimed to be the key factor of the ability of the KIRA compounds to attenuate RNase activity (44). The compounds were instead obtained as top hits from screening against the ATP binding structure (PDB ID 3P23), yet, as seen below, are nonetheless highly efficient in blocking RNase activity. This may be indicative of a mechanism of action of the KIRA compounds that is slightly more complex than that the induced distortion affecting oligomerization only.

To monitor the interaction between IRE1 and the compounds, we used microscale thermophoresis (MST) with recombinant IRE1 and increasing concentrations of Z4, Z4P (racemic mixture) and the pure Z4P enantiomers. We show that IRE1 interacts with both Z4 and Z4P following a saturation one-site binding curve exhibiting respective $\mathrm{K}_{\mathrm{D}}$ of $\sim 20$ and of $\sim 120 \mu \mathrm{M}$, respectively (Figure 2B). Since Z4P possesses a stereocenter, we next tested if both enantiomers would bind in the ATP-binding pocket of IRE1 and if they both do, which of them have a better affinity for the target. To this end, the racemic compound was synthesized and the enantiomers separated by chiral chromatography. Both enantiomers, named Z4P $(S)$ and Z4P $(R)$, were subjected to the same MST assay and were found to efficiently associate with IRE1 following a onesite saturation binding curves and with a marginal 2-fold difference in $\mathrm{K}_{\mathrm{D}}$, with $\sim 60$ and $\sim 29 \mu \mathrm{M}$, respectively (Figure S1). Additionally, all the Z4 family compounds were tested for inhibitory activity using a fluorescence resonance energy transfer (FRET) quenched IRE1 RNA substrate probe and yielded IC50s in the low micromolar range (Figures 2C and S2). IC50s of the Z4P (S) and Z4P (R) enantiomers were found to differ only by a 3-fold factor, highlighting again the rather unimportant nature of the stereocenter (Figure 2D).

\section{Z4 family molecules modulate IRE1 activity in vitro and in cellular models of GB}

- Having demonstrated that Z4 and Z4 derivatives (Z4A-E, Z4P) bind the IRE1 structure in silico and inhibit RNase activity of the human recombinant IRE1 protein in vitro, we proceeded to investigate whether these compounds could affect IRE1 activity in GB cells. Since the Z4 compounds bound the ATP kinase pocket in silico, we first documented their effect on IRE1 phosphorylation. Indeed, upon treatment with these compounds IRE1 phosphorylation was reduced by about 25\% (Figures 3A, S3A and S3B). Since kinase interference may mediate RNase effects (45) and since IRE1 has 
two distinct functional outputs through its RNase domain (XBP1 splicing and RIDD) we investigated the effect of Z4, Z4A-E and Z4P on Xbp1 mRNA splicing and RIDD mediated RNA decay. All Z4 family compounds inhibited Xbp1 mRNA splicing in U87 cells in the presence of the ER stressor tunicamycin (Figures 3B and S3C).
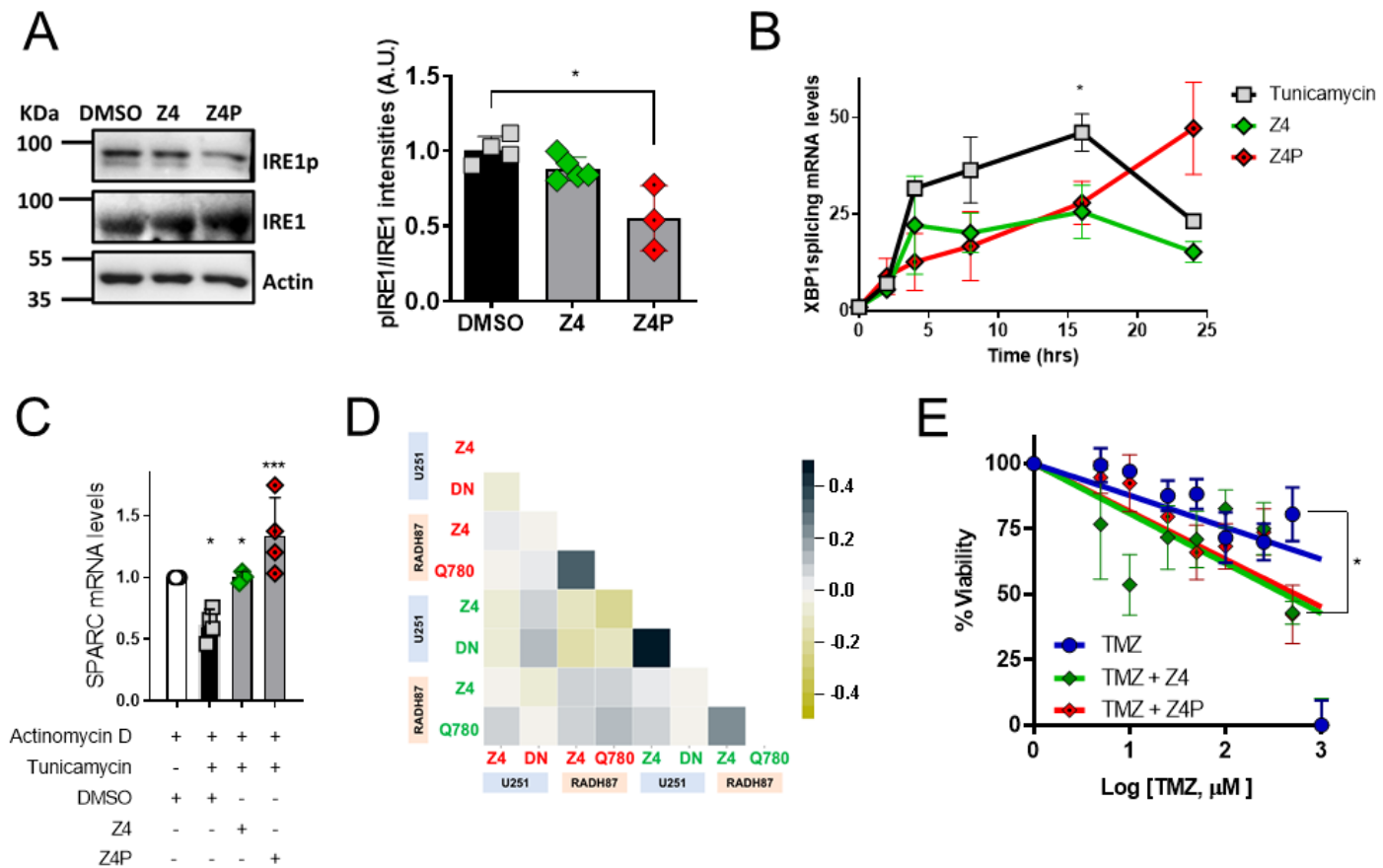

Figure 3: Z4 and Z4P inhibit IRE1 activity in cellular models. (A) Protein levels of IRE1 and phosphoIRE1 in U87 cells treated with Z4 and Z4P over 24 hours. Fold change of protein expression between IRE1 and phospho-IRE1 is represented in bar chart form normalized to untreated U87 cells. (B) Quantification of XBP1s mRNA levels upon treatment with $1 \mu \mathrm{g} / \mathrm{ml}$ Tunicamycin over 24hrs in the presence or absence of pre-treatments with $25 \mu \mathrm{M}$ Z4 or Z4P normalized to untreated U87 cells. (C) Quantification of SPARC mRNA levels in the presence of $5 \mu \mathrm{g} / \mathrm{ml}$ Actinomycin D and presence or absence of $10 \mu \mathrm{g} / \mathrm{ml}$ Tunicamycin and $9 \mu \mathrm{g} / \mathrm{ml}$ Z4 or Z4P over 4 hours in U87 cells. (D) Functional comparison of GB cell lines (RADH87 and U251) proteomes upon treatment with Z4 or in lines genetically impaired for IRE1 signaling. Red and green conditions remark annotations that were down or upregulated upon the respective conditions. (expressing a dominant-negative (DN) form of IRE1 or the Q780stop variant). (E) Cell viability of U87 cells as function of TMZ concentration, in the presence of increasing concentrations of Z4 and Z4P. The non-linear regression was calculated using the GraphPad Prism software. Symbols and error bars represent mean values $\pm S D$.

Having demonstrated the ability of Z4 compounds to block Xbp1 mRNA splicing, we next tested their effect on RIDD by interrogating the levels of the known RIDD target Sparc mRNA (46) in U87 cells upon treatment with the transcriptional blocker actinomycin D and the ER stressor tunicamycin. Both Z4 and Z4P blocked tunicamycin-induced degradation of Sparc mRNA compared to control treatment (Figure 3C) whilst had no effect on Sparc mRNA in cells overexpressing a dominant negative (DN) form of IRE1 (Figure S4A). We then investigated the specificity of these compounds in the context of the other arms of the UPR, PERK in particular. We did this to ensure that the Z4-compound related stress responses were wholly due to their 
action on IRE1 rather than other UPR transducers. We evaluated the expression of downstream effectors of PERK and ATF6. Neither Chop nor Herpud1 mRNA were significantly affected by Z4-compound treatment (Figure S4B-D). This was also confirmed at the level of elF2 $\alpha$ phosphorylation and ATF4 protein expression which were not altered upon exposure to Z4 (Figure S4B-D). These experiments gave us confidence that Z4 compounds did not significantly impact the other UPR branches. Consequently, to further document the characteristics of $Z 4$ at the protein level we treated U251 (commonly available GB cell line) and RADH87 (20) (human tumorderived primary GB cell line) cells and evaluated their proteome. Treatment of U251 and RADH87 cells with Z4 impacted on particular pathways such as cell adhesion, circulatory system development, cell locomotion and cell migration; all properties involved in tumor establishment (e.g. angiogenesis) and tumor invasion (e.g. metastasis) (Figure S5). We next functionally compared the proteomes of these GB lines exposed to $Z 4$ to those of the same cell lines in which IRE1 activity was invalidated genetically either using a dominant negative construct reported previously (21) or a truncated mutant variant of IRE1 lacking an RNase domain (Q780stop) (22) (Figure 3D and S5). Genes that were down- (red labels) or up-regulated (green labels) were compared according to the cell lines and conditions. Proteome alterations upon Z4 exposure phenocopied those observed in cells in which IRE1 activity was genetically blunted, thus reinforcing the selectivity of Z4 towards IRE1 (Figure 3D black squares). Having extensively documented the effect and specificity of Z4compounds on IRE1 we tested whether Z4 compounds could sensitize GB cells to the standard of care chemotherapy, TMZ. Cell viability assays were carried out on U87 cells treated with sub-toxic doses of Z4 and Z4P (data not shown) and escalating doses of TMZ. Both Z4 and Z4P produced similar effects, namely to significantly sensitize the cells to TMZ by about 1.5-fold (Figure 3E and S6). Our investigation so far has shown that (i) Z4P binds IRE1 in silico and in vitro; (ii) inhibits IRE1 activity in vitro and in cellular models; (iii) sensitizes GB cells to TMZ and; (iv) is predicted to cross the BBB. Collectively this meant that Z4P emerged as the most promising candidate for further investigations against $\mathrm{GB}$ in vivo.

\section{Combination therapy of Z4P and TMZ against in vivo models of GB arrests tumor} growth and prolongs relapse-free survival - To characterize Z4P in vivo, we utilized murine models growing orthotopic tumors generated by injection of U87-luc cells (25). 
First, we administered intraperitoneal (IP) escalating doses of Z4P (50 $\mu \mathrm{g} / \mathrm{kg}$ to 300 $\mu \mathrm{g} / \mathrm{kg}$ ) to determine Z4P toxicity in vivo. This was done in WT mice and no toxicity, assessed by weight loss, was detected in any of the concentrations tested (Figure S7A). Since Z4P did not display any toxicity in WT mice we then sought to (i) characterize the effect of Z4P on IRE1 biology in an in vivo tumor; (ii) test whether Z4P was able to cross the BBB and reach tumors cells; (iii) determine if co-treatment of Z4P alongside SOC chemotherapy TMZ conferred any anti-tumorigenic, anti-relapse or pro-survival advantages. To do so, U87-luc cells were orthotopically implanted in the mouse brain and the tumor was allowed to grow. At day 4 post implantation, a small tumor formation was detected using bioluminescence (data not shown). Thereafter, mice were randomized and Z4P daily treatments (i.p. $300 \mu \mathrm{g} / \mathrm{kg}$ ) were administered for 34 consecutive days (Figure 4A). Upon completion of this time period, mouse brains were entirely excised and dissected. The corresponding tumors were resected, dissociated and RNA extracted. The splicing of Xbp1 mRNA was evaluated in control tumors and in Z4P-treated animals. Treatment with Z4P decreased the splicing of Xbp1 mRNA thus demonstrating that Z4P mediates IRE1 inhibition in vivo when administered IP, also implying that it crossed the BBB (Figure 4B). Using the same mRNA samples, we also evaluated the expression levels of other GB invasion, inflammation and UPR markers using RT-qPCR (Figure 4C). A remarkable decrease of mRNA levels of genes related to inflammation (II-6 and II-8) whilst no expression difference could be seen in stemness genes (Cd133, Olig2, Sox2, Cd44 and Sall2) was observed. Vegfa mRNA, one of the genes related to vascular formation, had its levels decreased under Z4P treatment while Vimentin has a clear upregulation on Z4P treated group, results that were consistent with what was observed in U87 cells in which IRE1 activity was genetically invalidated $(21,47)$. The expression levels of Ecadherin (Cdh1) mRNA remained unchanged upon Z4P treatment. Lastly, apart from Bip, mRNA expression of the other ER stress markers tested (Chop, Herpud1 and Perk) decreased upon Z4P treatment. The latest results, although not identical to those obtained in cellular models might reflect a more complex mode of action of Z4P than expected (i.e. composition of the tumor stroma).

We next investigated the potential of Z4P as monotherapy or neo-adjuvant therapy alongside TMZ in a clinically relevant, surgical model of GB. Utilizing our Z4Ptreated, orthotopic mouse model (i.p. $300 \mu \mathrm{g} / \mathrm{kg}$ daily, starting on D4), we administered 10 daily treatments of TMZ (10 mg/kg; Figure 4D) after day 11-post tumor cell 
implantation. Whilst Z4P treatment alone had no effect on tumor growth or mouse survival (Figure S7B and S7C), co-treatment with TMZ significantly decreased tumor growth. This effect was observed as early as during the first week of treatment (Figure 4E and $4 F)$.

A

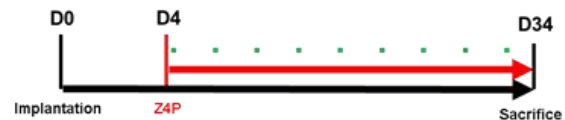

B

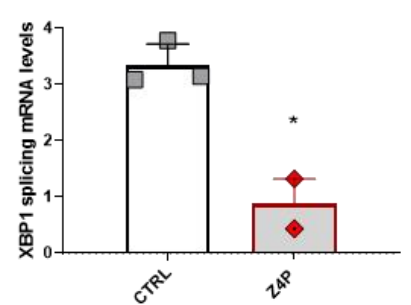

C

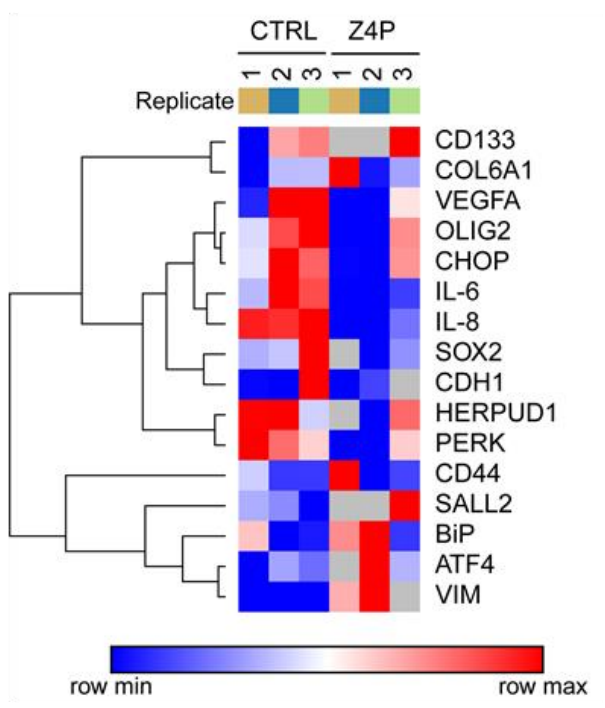

D

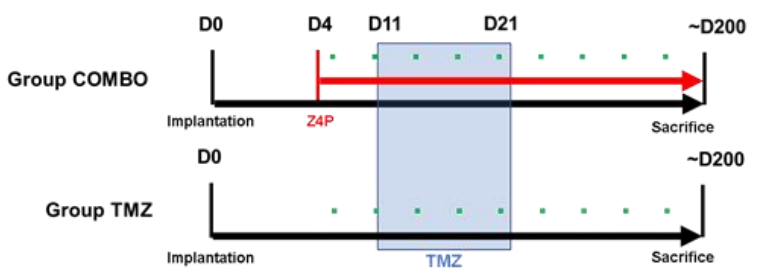

$E$

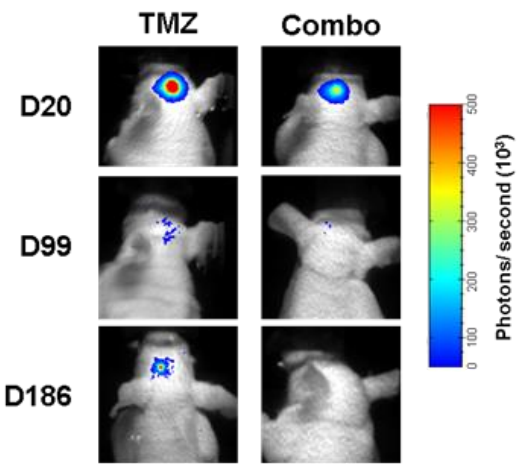

$\mathrm{F}$

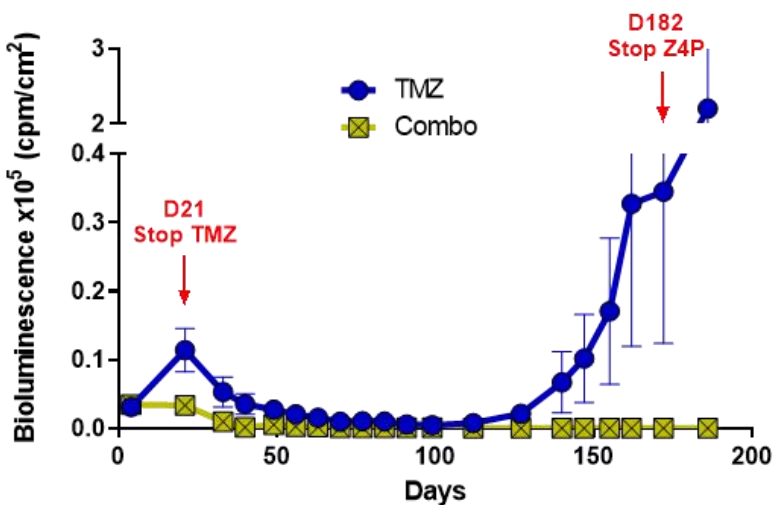

Figure 4: Z4P crosses the BBB, delays relapse in vivo under TMZ treatment. (A) Treatment timeline for Z4P (red) in GB model over 34 days. Period of tumor monitoring is expressed in green. (B) Quantification of XBP1s mRNA levels from in vivo tumors upon treatment with Z4P against the control. (C) mRNA levels analysis of UPR, stemness, vascular formation, migration and invasion markers for control and Z4 treated groups represented as heat map. Hierarchical clustering was performed by Morpheus online tool as described in the methods section (D) Treatment timeline for combination of Z4P (red) and TMZ (blue) in GB model over 186 days. Period of tumor monitoring is expressed in green (E) Tumor growth monitored by bioluminescence imaging. The bioluminescence images of the tumorbearing mice from the groups treated with $T M Z$ alone or in combination with Z4P (COMBO) at days 20 , 99 and 186 post-U87 cells implantation. (F) Quantitative analysis of bioluminescence imaging signal over 186 days of experiment for the groups treated with TMZ alone or in combination with Z4P (COMBO). Symbols and error bars represent mean values \pm SEM.

One of the aggressive hallmarks of clinical GB is the very high probability of tumor recurrence despite treatment with TMZ. To this end, we investigated whether co- 
treatment of Z4P and TMZ would have an effect on tumor recurrence. Over 200 days, expectedly, mice treated with TMZ alone relapsed (Figure 4F, blue curve) forming new tumors; a result consistent with what is observed in clinic. In contrast, no tumor relapse was observed in the group of animals treated with both TMZ and Z4P (Figure 4F, yellow curve); full panel of samples presented at D20 and D189 in Figure S8. In conclusion, Z4P, administered intraperitoneally, sensitizes orthotopically implanted GB tumors to $T M Z$ in mice. It prevents tumor relapse when used as an adjuvant therapy alongside TMZ. This has major translational clinical implications as GB tumor relapse is the major disease evolution characteristic causing death in human patients.

\section{Discussion}

Throughout tumor growth, tumor cells must cope with stresses such as nutrient shortage as well as therapeutic interventions that in some instances contribute to DNA damage (chemo and radiotherapy). The UPR and its sensor IRE1 have been identified as major actors in cancer cells adaptation to stress. As such, the pharmacological targeting of IRE1 in cancer represents an appealing therapeutic option. IRE1 modulators could be used as monotherapies or as adjuvant therapies alongside conventional or novel treatment regimes. The rationale behind using IRE1 inhibition in cancer is that by doing so, one can retard tumor growth by blocking the adaptive, prosurvival mechanisms employed by cancer cells against intrinsic stresses, or one can cripple the ability of cancer cells to cope with stresses such as those induced by chemotherapy or radiotherapy. IRE1 inhibitor monotherapies, or combination approaches with chemotherapeutic/antitumoral agents have been shown to significantly reduce tumor growth and impact metastasis and relapse in various preclinical cancer models $(2,6-8,12,13,15,16)$.

In glioblastoma (GB), the IRE1 RNase inhibitor MKC8866 was shown to enhance the efficacy of combined radiation and chemotherapy in vivo in mice (23). However, this molecule does not cross the BBB, requiring peri-surgical implantation of drug-containing biomaterials and limiting drug efficacy. No other IRE1 RNase $(4 \mu 8 \mathrm{c}$, HNA-based compounds, MKC family, among others) or kinase (KIRA family, GSK2850163) inhibitors have been shown to be able to efficiently cross the BBB. GB is a wholly fatal diagnosis and thus multiple novel therapeutic approaches including immunotherapy and oncolytic viruses have been investigated against it. However, 
response to such approaches is still limited, due to known mechanisms of resistance and tumor heterogeneity (48-51). With this in mind another attractive option could revolve around improving the efficacy of the standard of care (SOC) through adjuvant treatment with novel or repurposed compounds. Herein, we address this need by structurally interrogating the IRE1 tertiary structure. Specifically, by exploring the kinase catalytic domain through our established in silico pipeline (38), we identified a novel, not yet clinically approved, family of molecules (Z4-family) with different predicted specificities to established IRE1 kinase modulators, that inhibit IRE1 RNase activity in vitro and in vivo. Through the investigation of the ability of the Z4-family to modulate IRE1 RNase activity as well as their IRE1 binding specificity, we have confirmed that these molecules did not impact target genes of other arms of the UPR. Both, genetic- and Z4-mediated IRE1 inhibition impacted the proteomes of GB cells on functions related to metabolic and catabolic processes, cell adhesion and locomotion, essential hallmarks in cancer aggressiveness. In addition, the sensitization of GB cell lines under Z4-family treatment add a potential translational value for this molecule in clinic. We then tested our BBB crossing Z4-family candidate (Z4P) in an in vivo preclinical GB mouse model. The observation of a clear attenuation of Xbp1 mRNA splicing in Z4P treated tumors suggests the effective translocation of this drug through the BBB. However, more analyses should be done to effectively quantify the concentration of Z4P that can reach the tumor in vivo. We next, proceeded to co-treat mice with Z4P and TMZ. Despite there was no effect of Z4P alone, mice treated with a combination of Z4P and TMZ did not relapse after almost 200 days of treatment. Even with the limitation of no effect as a monotherapy, Z4P could be considered as a relevant adjuvant treatment option to extend the overall survival of GB patients. According to our ADMET data this molecule seems to be relatively unstable for long periods in aqueous solution which is also corroborated to the variability of the results after 24 hours incubation. In the in vivo assay, we bypassed this issue injecting multiple daily doses of Z4P during the experiment. However, studies that focus on StructureActivity relationship (SAR) could be extremely valuable to ameliorate this novel IRE1 inhibitor.

Our GB investigation focused on a particular member of the Z4-family (Z4P) because it could cross the BBB. However, other members of the Z4-family have even better in silico docking scores and in vitro $\mathrm{IC}_{50}$ than Z4P. This means that they could be investigated as adjuvant molecules in malignancies where the method of delivery 
is not an issue. We are thus enriching the landscape of IRE1 inhibitors available for investigation as adjuvant therapies alongside docetaxel/paclitaxel in breast cancer (13, 14), bortezomib in advanced multiple myeloma and acute myeloid leukemia $(7,8,11$, 13) as well as androgen deprivation therapies enzalutamide, abiraterone acetate, and chemotherapy cabazitaxel in prostate cancer models $(12,52)$. Further investigations are, however, needed to map the full repertoire of mechanisms by which Z4P-mediated IRE1 inhibition sensitize GB cells to chemotherapy. Studies have indicated that blocking Xbp1 mRNA splicing could reduce tumoral pro-survival adaptation against extrinsic stresses (15) whilst inhibition of RIDD may enhance the effects of DNA damaging agents (53). IRE1 is involved in multiple physiological and pathophysiological processes and its activation status varies from patient to patient. Indeed, we have reported upon an IRE1 activity signature that is able to stratify patient cohorts according to their XBP1 or RIDD activation status (20). Such a tool would be invaluable in determining which GB patients would benefit from IRE1 inhibition, thus enhancing the efficacy of molecules such as Z4P alongside SOC.

We have herein described a new family of compounds that inhibit IRE1 RNase activity by occupying its kinase ATP binding pocket. These compounds sensitize GB cells to TMZ-based chemotherapy in vitro. A member of this family (Z4P), can cross the BBB and we have shown that it inhibits IRE1 activity in orthotopic GB tumors in vivo when administered with TMZ. Importantly, cotreatment of GB tumors in vivo with Z4P and TMZ prevents tumor relapse, an effect observed upon TMZ treatment alone. The capability of Z4P to cross the BBB makes it an attractive potential therapeutic beyond GB. In addition, IRE1 signaling also plays important roles in pathological processes and mediates both adaptive and pro-apoptotic pathways in CNS diseases. Indeed, IRE1 signaling has been involved in a range of neurological disorders such as Parkinson's, Alzheimer's and Huntington's diseases, Amyotrophic Lateral Sclerosis, stroke and even infectious diseases such as cerebral malaria (54-60). To this end, genetic modulation of IRE1 signaling has been shown to prevent neurodegeneration (61). Our compound allows inhibition of IRE1 in CNS disease-relevant models, independent of their genetic manipulation. Our novel findings offer scope for further clinical investigation of the Z4 family in GB as well as other malignancies and provide opportunities for further characterization of IRE1 as an adjuvant therapy in oncology and beyond. 


\section{Acknowledgements}

We thank Drs F. Delom, D. Fessart, D. Gilot, E. Lafont, E. Ogier-Denis as well as Prs A. Samali and R. Gillet, for the critical reading of the manuscript. Part of this work was carried out using the Spectroscopies-CDTP core facility and the animal facility ARCHE (UMS Biosit, Université de Rennes 1- Campus de Villejean - 35043 RENNES Cedex, FRANCE). This work was supported by grants from Institut National du Cancer (INCa; PLBIO), Agence Nationale de la Recherche (ANR; ERAAT), Fondation pour la Recherche Médicale (FRM; DEQ20180339169) and Institut des Neurosciences Cliniques de Rennes (INCR) to EC; EU H2020 MSCA ITN-675448 (TRAINERS) and MSCA RISE-734749 (INSPIRED) grants to EC, AC, and LAE; la Ligue contre le Cancer (comités 35, 56 et 85) to TA. The Swedish Research Council (VR; grant no 2019-3684) and the Swedish National Infrastructure for Computing (SNIC; funded in part through VR grant agreement 2018-05973) are gratefully acknowledged for funding and allocations of computing time, respectively (LAE). DPR was funded by grants from INSERM (La Vannetaise) and the Brittany Region, DD was funded by EU H2020 MSCA ITN-675448, XG was funded by the Fondation ARC pour la recherche sur le cancer (PDF20191209830), CS was funded by a post-doctoral fellowship from INSERM-plan cancer.

\section{Conflict of Interest}

LEA and EC are founders of Cell Stress Discoveries Ltd. AC is a founder of e-NIOS Applications PC (https://e-nios.com/).

\section{Author contribution}

DPR, DD, XG, RP - conceptualization, methodology, investigation; TK, CS, LN methodology, investigation; AC, EC, LEA, TA - supervision, conceptualization, project administration, funding acquisition; writing (https://www.casrai.org/credit.html\#).

\section{References}

1. C. Hetz, J. M. Axten, J. B. Patterson, Publisher Correction: Pharmacological targeting of the unfolded protein response for disease intervention. Nat Chem Biol 15, 1129 (2019).

2. D. P. Raymundo, et al., Pharmacological Targeting of IRE1 in Cancer. Trends in Cancer 6, 10181030 (2020).

3. L. Wang, et al., Divergent allosteric control of the IRE1 $\alpha$ endoribonuclease using kinase inhibitors (2012). 
4. H. C. Feldman, et al., Structural and Functional Analysis of the Allosteric Inhibition of IRE1a with ATP-Competitive Ligands. ACS Chemical Biology 11, 2195-2205 (2016).

5. C. Hetz, E. Chevet, H. P. Harding, Targeting the unfolded protein response in disease. Nature Reviews Drug Discovery 12, 703-19 (2013).

6. J. Ming, et al., A novel chemical, STF-083010, reverses tamoxifen-related drug resistance in breast cancer by inhibiting IRE1/XBP1. Oncotarget 6, 40692-40703 (2015).

7. M. Ri, et al., Identification of Toyocamycin, an agent cytotoxic for multiple myeloma cells, as a potent inhibitor of ER stress-induced XBP1 mRNA splicing. Blood Cancer Journal 2, e79-e79 (2012).

8. N. Mimura, et al., Blockade of XBP1 splicing by inhibition of IRE1a is a promising therapeutic option in multiple myeloma. Blood 119, 5772-5781 (2012).

9. B. C. S. Cross, et al., The molecular basis for selective inhibition of unconventional mRNA splicing by an IRE1-binding small molecule. Proc Natl Acad Sci U S A 109, E869-878 (2012).

10. K. L. Kemp, et al., The serine-threonine kinase inositol-requiring enzyme 1a (IRE1 $\alpha$ ) promotes IL4 production in T helper cells. J Biol Chem 288, 33272-33282 (2013).

11. A. Palumbo, et al., Bortezomib, doxorubicin and dexamethasone in advanced multiple myeloma. Annals of Oncology 19, 1160-1165 (2008).

12. X. Sheng, et al., IRE1a-XBP1s pathway promotes prostate cancer by activating c-MYC signaling. Nature Communications 10, 323 (2019).

13. H. Sun, et al., Inhibition of IRE1a-driven pro-survival pathways is a promising therapeutic application in acute myeloid leukemia. Oncotarget 7, 18736-18749 (2016).

14. C. L. Kriss, et al., Overexpression of TCL1 activates the endoplasmic reticulum stress response: a novel mechanism of leukemic progression in mice. Blood 120, 1027-1038 (2012).

15. J. M. Harnoss, et al., IRE1a Disruption in Triple-Negative Breast Cancer Cooperates with Antiangiogenic Therapy by Reversing ER Stress Adaptation and Remodeling the Tumor Microenvironment. Cancer Res 80, 2368-2379 (2020).

16. S. E. Logue, et al., Inhibition of IRE1 RNase activity modulates the tumor cell secretome and enhances response to chemotherapy. Nature Communications 9 (2018).

17. R. Stupp, et al., Radiotherapy plus concomitant and adjuvant temozolomide for glioblastoma. $N$ Engl J Med 352, 987-996 (2005).

18. D. Doultsinos, et al., Control of the Unfolded Protein Response in Health and Disease. SLAS Discov 22, 787-800 (2017).

19. J. Obacz, et al., Endoplasmic reticulum proteostasis in glioblastoma-From molecular mechanisms to therapeutic perspectives. Science Signaling 10 (2017).

20. A. G, et al., Inositol-requiring enzyme 1alpha is a key regulator of angiogenesis and invasion in malignant glioma. Proc Natl Acad Sci U S A 107, 15553-15558 (2010).

21. B. Drogat, et al., IRE1 signaling is essential for ischemia-induced vascular endothelial growth factor-A expression and contributes to angiogenesis and tumor growth in vivo. Cancer Res 67, 6700-6707 (2007).

22. S. Lhomond, et al., Dual IRE1 RNase functions dictate glioblastoma development. EMBO Molecular Medicine 10, e7929 (2018).

23. P. J. Le Reste, et al., Local intracerebral inhibition of IRE1 by MKC8866 sensitizes glioblastoma to irradiation/chemotherapy in vivo. Cancer Letters 494, 73-83 (2020).

24. A. Goede, E. Michalsky, U. Schmidt, R. Preissner, SuperMimic--fitting peptide mimetics into protein structures. BMC bioinformatics 7, 11 (2006).

25. E. F. Pettersen, et al., UCSF Chimera - A visualization system for exploratory research and analysis. Journal of Computational Chemistry 25, 1605-1612 (2004).

26. A. Daina, O. Michielin, V. Zoete, SwissADME: a free web tool to evaluate pharmacokinetics, druglikeness and medicinal chemistry friendliness of small molecules. Scientific reports 7, 42717 (2017)

27. A. Daina, V. Zoete, A BOILED-Egg To Predict Gastrointestinal Absorption and Brain Penetration of Small Molecules. ChemMedChem 11, 1117-1121 (2016).

28. F. Prischi, P. R. Nowak, M. Carrara, M. M. U. Ali, Phosphoregulation of Ire1 RNase splicing activity. Nature Communications 5, 3554 (2014).

29. T. Avril, et al., Human glioblastoma stem-like cells are more sensitive to allogeneic NK and T cellmediated killing compared with serum-cultured glioblastoma cells. Brain Pathol 22, 159-174 (2012).

30. J. Obacz, et al., Novel IRE1-dependent proinflammatory signaling controls tumor infiltration by myeloid cells. bioRxiv, 533018 (2020). 
31. S. Lhomond, et al., Adaptation of the secretory pathway in cancer through IRE1 signaling. Methods Mol Biol 1292, 177-194 (2015).

32. F. Hong, et al., RankProd: a bioconductor package for detecting differentially expressed genes in meta-analysis. Bioinformatics 22, 2825-2827 (2006).

33. T. Koutsandreas, et al., Analyzing and Visualizing Genomic Complexity for the Derivation of the Emergent Molecular Networks. Int. J. Monit. Surveill. Technol. Res. 4, 30-49 (2016).

34. , https://bioinfominer.com/ (e-NIOS Applications P.C.).

35. The Gene Ontology Consortium, The Gene Ontology resource: enriching a GOld mine. Nucleic Acids Research 49, D325-D334 (2021).

36. P. Resnik, Semantic Similarity in a Taxonomy: An Information-Based Measure and its Application to Problems of Ambiguity in Natural Language. Journal of Artificial Intelligence Research 11, 95130 (1999).

37. A. Jabouille, et al., Glioblastoma invasion and cooption depend on IRE1 $\alpha$ endoribonuclease activity. Oncotarget 6, 24922-24934 (2015).

38. D. Doultsinos, et al., Peptidomimetic-based identification of FDA-approved compounds inhibiting IRE1 activity. The FEBS Journal 288, 945-960 (2021).

39. T. Sterling, J. J. Irwin, ZINC 15--Ligand Discovery for Everyone. Journal of chemical information and modeling 55, 2324-2337 (2015).

40. S. Lhomond, et al., Dual IRE1 RNase functions dictate glioblastoma development. EMBO Mol Med 10 (2018).

41. P. Willett, Similarity-based virtual screening using 2D fingerprints. Drug Discovery Today 11, 1046-1053 (2006).

42. R. A. Friesner, et al., Extra Precision Glide: Docking and Scoring Incorporating a Model of Hydrophobic Enclosure for Protein-Ligand Complexes. Journal of Medicinal Chemistry 49, 61776196 (2006).

43. J. L. Banks, et al., Integrated Modeling Program, Applied Chemical Theory (IMPACT). Journal of computational chemistry 26, 1752-1780 (2005).

44. D. Korovesis, N. Rufo, R. Derua, P. Agostinis, S. H. L. Verhelst, Kinase Photoaffinity Labeling Reveals Low Selectivity Profile of the IRE1 Targeting Imidazopyrazine-Based KIRA6 Inhibitor. ACS Chem. Biol. 15, 3106-3111 (2020).

45. W. Tirasophon, A. A. Welihinda, R. J. Kaufman, A stress response pathway from the endoplasmic reticulum to the nucleus requires a novel bifunctional protein kinase/endoribonuclease (Ire1p) in mammalian cells. Genes Dev. 12, 1812-1824 (1998).

46. N. Dejeans, et al., Autocrine control of glioma cells adhesion and migration through IRE1alphamediated cleavage of SPARC mRNA. J Cell Sci 125, 4278-4287 (2012).

47. G. Auf, et al., Inositol-requiring enzyme 1 is a key regulator of angiogenesis and invasion in malignant glioma. Proceedings of the National Academy of Sciences 107, 15553-15558 (2010).

48. C. M. Jackson, J. Choi, M. Lim, Mechanisms of immunotherapy resistance: lessons from glioblastoma. Nature Immunology 20, 1100-1109 (2019).

49. R. Medikonda, G. Dunn, M. Rahman, P. Fecci, M. Lim, A review of glioblastoma immunotherapy. J Neurooncol 151, 41-53 (2021).

50. D. S. Kim, Cancer stem cell plasticity in glioblastoma multiforme: a perspective on future directions in oncolytic virotherapy. Future Oncol 16, 2251-2264 (2020).

51. V. M. Lu, et al., Clinical trials using oncolytic viral therapy to treat adult glioblastoma: a progress report. Neurosurg Focus 50, E3 (2021).

52. X. Sheng, et al., Divergent androgen regulation of unfolded protein response pathways drives prostate cancer. EMBO Mol Med 7, 788-801 (2015).

53. E. Dufey, et al., Genotoxic stress triggers the activation of IRE1a-dependent RNA decay to modulate the DNA damage response. Nat Commun 11, 2401 (2020).

54. S. Xu, et al., Mesencephalic astrocyte-derived neurotrophic factor (MANF) protects against $A \beta$ toxicity via attenuating $A \beta$-induced endoplasmic reticulum stress. $J$ Neuroinflammation 16, 35 (2019).

55. H. Ni, Q. Rui, D. Li, R. Gao, G. Chen, The Role of IRE1 Signaling in the Central Nervous System Diseases. Curr Neuropharmacol 16, 1340-1347 (2018).

56. C. Yan, et al., IRE1 promotes neurodegeneration through autophagy-dependent neuron death in the Drosophila model of Parkinson's disease. Cell Death \& Disease 10, 1-15 (2019).

57. C. Xiang, Y. Wang, H. Zhang, F. Han, The role of endoplasmic reticulum stress in neurodegenerative disease. Apoptosis 22, 1-26 (2017).

58. D. B. Medinas, J. V. González, P. Falcon, C. Hetz, Fine-Tuning ER Stress Signal Transducers to Treat Amyotrophic Lateral Sclerosis. Front. Mol. Neurosci. 10 (2017). 
59. E. Kiskinis, et al., Pathways disrupted in human ALS motor neurons identified through genetic correction of mutant SOD1. Cell Stem Cell 14, 781-795 (2014).

60. C. Duran-Aniotz, et al., IRE1 signaling exacerbates Alzheimer's disease pathogenesis. Acta Neuropathol 134, 489-506 (2017).

61. J. M. D. Grandjean, et al., Pharmacologic IRE1/XBP1s activation confers targeted ER proteostasis reprogramming. Nat Chem Biol 16, 1052-1061 (2020).

62. M. M. Ali, et al., Structure of the Ire1 autophosphorylation complex and implications for the unfolded protein response. EMBO J 30, 894-905 (2011).

63. P. E. Harrington, et al., Unfolded protein response in cancer: IRE1 $\alpha$ inhibition by selective kinase ligands does not impair tumor cell viability. ACS Medicinal Chemistry Letters 6, 68-72 (2015). 